\title{
Effect of non-toxic, filamentous cyanobacteria on egg abortion in Daphnia under various thermal conditions
}

\author{
Anna Bednarska $\cdot$ Miroslaw Slusarczyk
}

Received: 30 March 2012 / Accepted: 1 December 2012/Published online: 29 December 2012

(C) The Author(s) 2012. This article is published with open access at Springerlink.com

\begin{abstract}
The aim of this study was to examine whether the presence of non-toxic filamentous cyanobacteria can cause egg abortion in Daphnia. To test this hypothesis, we fed females of Daphnia pulicaria with the green alga Scenedesmus obliquus from birth until eggs were laid in the brood chamber. Gravid Daphnia females were then exposed to media containing either S. obliquus or filaments of a non-toxic strain of the cyanobacterium Cylindrospermopsis raciborskii. The females were then observed throughout the whole period of egg development and all events of egg abortion were recorded. Experiments were conducted at three temperatures: 16,20 , and $24^{\circ} \mathrm{C}$. In the presence of cyanobacteria, the proportion of aborted eggs ranged from 2.6 to $12.9 \%$, compared with $0-1.8 \%$ in the green algae treatment. The number of aborted eggs, but not the number of egg-aborting females, tended to increase at higher temperature. Our data indicate that interference by non-toxic cyanobacterial filaments may affect the reproductive success of Daphnia, not only through suppression of their fecundity, but also via an increase in the number of aborted eggs. Therefore, the reproductive rate of Daphnia based on the fecundity of
\end{abstract}

Guest editors: Marina Manca \& Piet Spaak / Cladocera: Proceedings of the 9th International Symposium on Cladocera

A. Bednarska $(\bowtie) \cdot$ M. Slusarczyk Department of Hydrobiology, University of Warsaw, Banacha 2, 02-097 Warsaw, Poland e-mail: a.bednarska@uw.edu.pl females may be overestimated in situations where filamentous cyanobacteria are present.

Keywords Daphnia-cyanobacteria interactions · Temperature $\cdot$ Reproductive success

\section{Introduction}

Cyanobacteria are an inadequate food source for zooplankton (Lampert, 1987; deBernardi \& Giussani, 1990). They may negatively affect the fitness of herbivorous planktonic animals through (i) toxicity of their secondary metabolites-hepato- and neurotoxins (e.g., Wilson et al., 2006), (ii) poor nutritional quality - the lack of sufficient amount of compounds such as polyunsaturated fatty acids (PUFAs) and sterols that are essential for growth and reproduction of crustaceans (e.g., Von Elert, 2004; Martin-Creuzburg et al., 2008), or (iii) poor manageabilityinterference of cyanobacterial filaments or colonies with the food gathering process (e.g., Gliwicz \& Siedlar, 1980; Abrusán, 2004).

Non-selective planktonic animals cannot avoid feeding on cyanobacteria and large-bodied herbivorous cladocerans of the genus Daphnia are particularly affected by their presence. The dominance of cyanobacteria among phytoplankton often leads to a reduction in Daphnia abundance within a zooplankton community (Kirk \& Gilbert, 1992; Ghadouani et al., 2003), and the presence of cyanobacteria can result in 
delayed reproduction, decreases in body size, fecundity, and growth rate, and shortened lifespan (see review e.g. Porter \& Orcutt, 1980; Lampert, 1987; deBernardi \& Giussani, 1990). Of the phenotypic traits of Daphnia that are negatively affected by cyanobacteria, the reproduction seems to be most sensitive. Toxic cyanobacteria may affect Daphnia reproduction not only by suppressing its fecundity but also by increasing the number of aborted eggs or causing egg/ embryo malformation and/or decomposition (Reinikainen et al., 1995; Claska \& Gilbert, 1998; Gustafsson et al., 2005; Dao et al., 2010). Reinikainen et al. (1995) suggested that cyanotoxins can disturb the development of Daphnia eggs and embryos. They also proposed that these toxins could be transferred from mothers to eggs. Microcystins are cyanotoxins that have lipophilic properties and can be ingested by Daphnia, accumulated in their lipids, and thus transferred from mother to progeny during egg formation. The abortion of eggs in the presence of toxic M. aeruginosa was reported by Gustafsson et al. (2005) and Dao et al. (2010). Furthermore, the latter study showed that toxic cyanobacteria can cause not only egg abortion/decomposition in Daphnia but also malformation of neonates. The production of eggs that fail to develop into viable offspring is a wasted investment, especially when fecundity is decreased on a cyanobacteria diet and the acquisition of the necessary compounds for egg formation (like PUFAs and sterols) is difficult. Under nutritional limitation and in the absence of sufficient amounts of essential compounds, eggs may degenerate and offspring fail to develop (Brooks, 1946). Thus, the poor quality of cyanobacteria as the available food source could be responsible for egg abortion or malformation although there is little evidence in the literature to support this claim. Some indirect evidence derived from field studies indicates that the poor quality of cyanobacteria can induce egg degeneration. For example, Redfield (1981) identified a positive relationship between the proportion of degenerated eggs in Daphnia and the level of ungrazable phytoplankton. Moreover, Threlkeld (1979) found that egg degeneration occurred when the presence of cyanobacteria was combined with higher temperature.

Our observations of Daphnia kept in the presence of non-toxic filamentous cyanobacteria have suggested that another cause of egg abortion, besides toxicity or the poor quality of cyanobacteria, is the limited capacity of Daphnia to handle cyanobacterial filaments.

For the purpose of this study, we will use the term "abortion" for all mechanisms leading to increased egg mortality in the brood chamber during the period of egg development.

The presence of filamentous or colonial cyanobacteria in the environment may cause mechanical interference with the food-gathering process of Daphnia and trigger frequent cleaning movements of the postabdominal claws (Gliwicz \& Siedlar, 1980). During such cleaning movements, the abdomen moves upwards and then outwards and the postabdominal claws remove any interfering particles from the feeding apparatus and subsequently the filtering chamber. If interference by cyanobacterial filaments is extensive, the abdominal movements become intense and "erratic." During such movements, the first abdominal process (a structure in the lower rear part of the abdomen, Fig. 1), which normally "closes" the bottom part of the brood chamber, also moves upwards. Our observations suggest that during such movements (i) eggs can fall out of the "opened" brood chamber or (ii) the first abdominal process may damage the egg membrane causing its disintegration as the postabdomen moves backwards (personal observations).

The negative effect of cyanobacteria on Daphnia life history is positively correlated with temperature

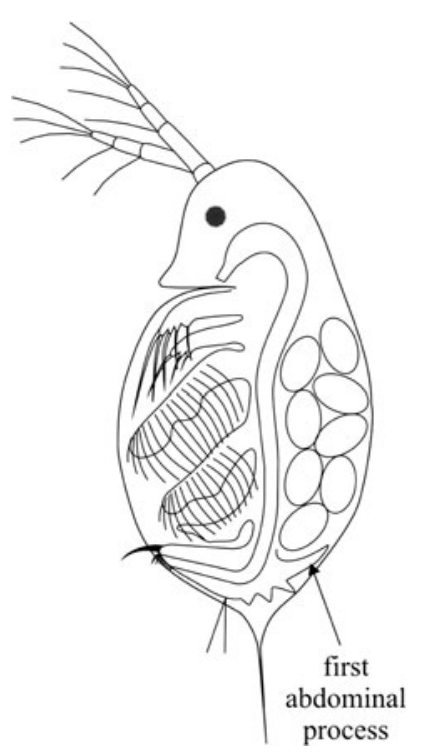

Fig. 1 Gravid Daphnia female with the first abdominal process visible 
(Threlkeld, 1979; Claska \& Gilbert, 1998; Abrusán, 2004; Sperfeld \& Wacker, 2009; Bednarska et al., 2011). The level of interference with the foodgathering process caused by cyanobacteria also increases with rising temperature (see Bednarska, 2006, for a review on the mechanism of food retention in Daphnia). Thus, we hypothesized that the extent of egg abortion in the presence of cyanobacteria may depend on the thermal conditions.

This study was designed to examine whether the presence of non-toxic filamentous cyanobacteria can cause egg abortion in Daphnia. Female Daphnia pulicaria were fed with the green alga Scenedesmus obliquus from birth until eggs were laid in the brood chambers (thus, all substances required for proper development of the eggs were present in the food during the period of egg formation). The egg-bearing females were then exposed either to S. obliquus, or to the non-toxic filamentous cyanobacterium Cylindrospermopsis raciborskii. Experiments were performed at three different temperatures with two clones of Daphnia. To our knowledge, this is the first study that has produced evidence for the phenomenon of egg abortion in Daphnia stimulated by the presence of non-toxic filamentous cyanobacteria.

\section{Methods}

Experiments were performed with two clones of D. pulicaria Forbes $(\mathrm{Br} 1$ and $\mathrm{Br} 2)$ originating from the eutrophic lake Brome in Quebec, Canada. The clones were hatched from ephippia in our laboratory and maintained under standard conditions [i.e., batch cultures at room temperature $\left(19-22^{\circ} \mathrm{C}\right)$, fed with the green alga $S$. obliquus - SAG 276-3a] for at least 1 year before the study.

Clonal lineages used in the experiments were established by selecting a single female from each stock culture. The animals were further pre-cultured for at least three generations under standard conditions, i.e., in a temperature-controlled water bath $\left(20 \pm 0.5^{\circ} \mathrm{C}\right)$, with a summer photoperiod (16L:8D), fed daily with S. obliquus (1 $\mathrm{mg} \mathrm{C}^{-1}$ ), changing the culture medium every other day. This procedure was employed to minimize the potential variation between clones caused by differences in the culture conditions of the mothers.

Conditioned lake water (water from a small eutrophic pond Szczęśliwice located in Warsaw, Poland, prefiltered through a $1-\mu \mathrm{m}$ filter, and stored in a large aerated tank for 2 weeks before use) was used to prepare all media. Before preparing the experimental media, the water was filtered again through a $0.2-\mu \mathrm{m}$ filter and enriched with suspensions of either the green alga $S$. obliquus or filaments of the non-toxic cyanobacterium C. raciborskii (strain SAG 1.97) as the food source. The lack of toxin production by this strain was confirmed by HPLC with diode array detection (Bednarska, unpublished data). Also, no signs of toxicity were detected in a standard toxicity test with Daphnia conducted before the experiment (Bednarska, unpublished data). Both the green algae and the cyanobacteria were cultured in batch cultures on Z/4 medium (Zehnder \& Gorham, 1960), at room temperature $\left(20 \pm 0.5^{\circ} \mathrm{C}\right)$, under constant illumination with aeration.

Synchronized cohorts (born within 10 h) of Daphnia females used in the experiments were fed with moderate levels of $S$. obliquus $\left(0.65 \mathrm{mg} \mathrm{C}^{-1}\right)$ until eggs had been produced and laid in the brood chamber. The females were reared on this green alga to insure the presence of all essential substances for the production and normal development of eggs. The food concentration was slightly restricted to limit egg production so that the number laid in the brood chamber could be determined reliably. The experimental females were held in a temperature-controlled water bath $\left(20 \pm 0.5^{\circ} \mathrm{C}\right)$, under a summer photoperiod (16L:8D), and the culture medium was changed daily.

The experiments themselves were conducted with primiparous females and started within $12 \mathrm{~h}$ after the eggs had been laid in the brood chambers. Females carrying 5-8 eggs were randomly assigned to two different food (1 mg C $1^{-1}$ of $S$. obliquus or $1 \mathrm{mg}$ $\mathrm{C}^{-1}$ of C. raciborskii) and three different temperature $\left(16,20\right.$, or $\left.24^{\circ} \mathrm{C}\right)$ treatments. As required, the temperature was lowered to $16^{\circ} \mathrm{C}$ or raised to $24^{\circ} \mathrm{C}$ (from the starting temperature of $20^{\circ} \mathrm{C}$ ) over the course of $6 \mathrm{~h}$ to avoid stressing the Daphnia. The experiments were performed with a factorial design: two food treatments, two clones, and three temperatures. All animals were maintained individually in 35-ml, V-bottomed vessels, and there were ten replicates for each of the six treatments. V-bottomed vessels were used to more easily count and collect eggs if they had been lost from the brood chamber. The number of eggs/embryos in the brood chamber of each female was checked daily until the newborns were 
released. The number of viable offspring was recorded. The experiment conducted at 16,20 , or $24^{\circ} \mathrm{C}$ lasted $4.7 \pm 0.5 ; 3.1 \pm 0.3$; or $2.1 \pm 0.3$ days on average ( $\pm 1 \mathrm{SD})$, respectively.

Statistical analysis of the collected data was performed by means of Statistica 9 software. The effect of food (green algae vs. filamentous cyanobacteria) and clone as treatment factors, and environmental temperature as a continuous variable, on the proportion of aborting females among the ten individuals tested in each of the 12 experimental treatments was verified in the generalized linear model with binary response variable, binomial error distribution, and $\log -\log$ link function. The effect of the same factors and variables on the proportion of aborted eggs among those that were formed by each experimental female was tested in the generalized linear model with binomial error distribution and logit link function.

\section{Results}

The food type during the period of egg bearing had a significant effect on the proportion of aborting females (GLZ, df $\left.=1,115, \chi^{2}=13.6, P=0.0002\right)$. In the green algae treatments, all but one female (98\%) released all offspring developing from eggs laid in the brood chambers; whereas in the cyanobacteria treatments, the proportion of non-aborting females ranged from 70 to $90 \%$ with a mean of $78.3 \%$. Neither temperature $\left(\mathrm{GLZ}, \mathrm{df}=1,115, \chi^{2}=0.48, P=0.48\right.$ ) nor clone identity (GLZ, df $=1,115, \chi^{2}=1.4$, $P=0.24)$ or clone-food interaction (GLZ, $\mathrm{df}=1$, $115, \chi^{2}=0.89, P=0.34$ ) had a significant effect on the proportion of aborting females.

However, the food type during the period of egg bearing (GLZ, df $=1,115, \chi^{2}=24.1, P=0.000001$ ) (Figs. 2, 3) and the temperature (GLZ, df $=1,115$, $\left.\chi^{2}=5.2, P<0.022\right)$ did have a significant effect on the proportion of aborted eggs. Only one egg was aborted in the green algae treatments $(0.3 \%$ on average for all green algae treatments); whereas in the cyanobacteria treatments, the proportion of aborted eggs was significantly higher and ranged (mean for two clones) from $9.5 \%$ at $24^{\circ} \mathrm{C}, 6.3 \%$ at $20^{\circ} \mathrm{C}$, to $3.3 \%$ at $16^{\circ} \mathrm{C}$ (mean of $6.4 \%$ for all cyanobacteria treatments). Neither clonal affiliation (GLZ, df $=1,115, \chi^{2}=$ $1.17, P=0.28$ ) nor interaction between clonal identity or the food type (GLZ, df $=1,115, \chi^{2}=0.89$,

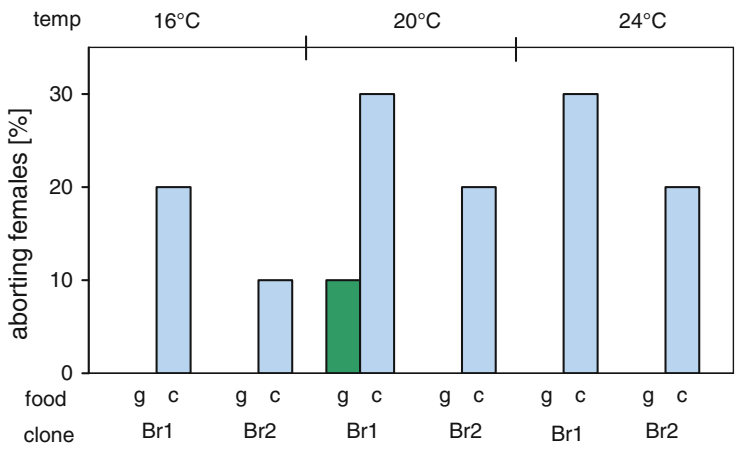

Fig. 2 The proportion of aborting females in two clones of D. pulicaria $(\mathrm{Br} 1$ and $\mathrm{Br} 2)$ kept at different thermal conditions with or without cyanobacteria ( $c$ cyanobacteria, $g$ green algae)

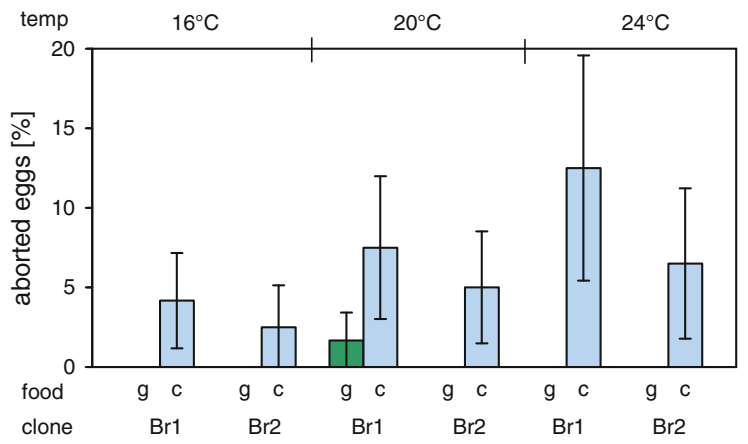

Fig. 3 The proportion (mean $\pm 1 \mathrm{SE}$ ) of aborted eggs by females of two clones of $D$. pulicaria $(\mathrm{Br} 1$ and $\mathrm{Br} 2)$ kept at different thermal conditions with or without cyanobacteria ( $c$ cyanobacteria, $g$ green algae)

$P=0.34)$ produced a significant effect on the proportion of aborted eggs.

\section{Discussion}

The aim of this study was to examine whether the presence of non-toxic filaments of cyanobacteria may lead to egg abortion in Daphnia, and if this can be associated solely with the poor capacity of Daphnia to handle cyanobacterial filaments. Our results indicate that indeed the presence of non-toxic filamentous cyanobacteria can cause abortion of eggs. The experimental protocol, in which Daphnia were fed a green alga until eggs were laid in the brood chamber (i.e., during the period of eggs formation) and only then were they exposed to the presence of cyanobacteria, allowed us to rule out the possibility that any egg abortion was caused by the poor nutritional properties 
of cyanobacteria. The use of a non-toxic cyanobacterial strain also excluded any complicating toxic effects. It is rather unlikely that the disappearance of eggs from the brood chambers was the result of spontaneous degradation caused by poor egg nutrition. First, the process of self-degradation of Daphnia eggs proceeds gradually (Brooks, 1946) and both correctly developing embryos and degenerating eggs can occur in the brood chambers simultaneously (Brooks, 1946), but this was not observed upon daily inspection of the gravid females in our study. Second, the females before the deposition of eggs into the brood chamber were fed with a high concentration (above the incipient food level) of the edible alga $S$. obliquus (a high quality food commonly used in Daphnia studies), so no nutritional limitation should occur. In this case, all eggs present in the brood chambers progressed normally through all stages of development and there was no sign of egg degradation. Previously, Repka (1996) compared the egg mortality in Daphnia fed with the green alga S. obliquus and those fed the filamentous cyanobacterium Oscillatoria limnetica and found higher egg mortality in the former. This seems to contradict our findings, but a crucial difference in the experimental design (in our study all eggs were produced by Scenedesmus-fed mothers) makes it hard to compare the results of these two studies. In addition, Repka (1996) suggested that increased egg mortality in Scenedesmus-fed animals might have been caused by changes in the quality of the green algae used.

In this study, all eggs were produced by Scenedes$m u s$-fed mothers and it is unlikely that the food type offered to mothers during the period of egg bearing affected the nutrition of eggs which had already been laid into the brood chamber. However, the presence of cyanobacteria in the medium could be stressful for the mothers; thus, one can argue that egg abortion observed in the study was caused by egg resorption by food-limited mothers. Unfertilized ephippial eggs can be resorbed by Daphnia, but only when eggs are still in the ovaries (Zaffagnini, 1987). We did not find any reliable information concerning the possibility of eggs resorption from the brood chamber in the literature, and this seems rather unlikely. We are unaware whether destructed eggs can serve as a food source (like detritus particles) for mothers and whether they could compensate for food deficiencies in cyanobacteria treatments. This would rather be a costly and ineffective way of compensation of nutritional limitation, and while we doubt it, we can not rule it out completely.

We found no significant differences in the proportion of aborting females or that of aborted eggs between the two studied Daphnia clones. The mean proportions of aborting females $(21.7 \%)$ and aborted eggs $(6.4 \%)$ observed in the cyanobacteria treatment groups were lower than those previously reported for Daphnia fed on toxic cyanobacteria (Reinikainen et al., 1995; Dao et al., 2010). This may have been due to the exclusion of possible chemical side effects in the present study so that any influence of the non-toxic cyanobacteria on egg abortion was purely mechanical.

It has previously been shown that the negative effect of cyanobacteria on Daphnia performance can be more pronounced at higher temperature. For example, raised sensitivity to cyanotoxin at higher temperature was reported by Claska \& Gilbert (1998). Sperfeld \& Wacker (2009) found greater sterol limitation in Daphnia fed with cyanobacteria at higher temperature due to the increased demand for these compounds. Temperature affects water viscosity, and so a higher temperature may also intensify the disturbance of the food gathering process caused by the presence of filamentous cyanobacteria (Abrusán, 2004, also see Bednarska, 2006, for a review of the mechanism). It has been confirmed both in in situ experiments (Threlkeld, 1979) and under laboratory conditions (Bednarska et al., 2011) that feeding on cyanobacteria at raised temperatures can decrease the fecundity of Daphnia. In addition, as reported in this study, the number of aborted eggs in the presence of cyanobacteria appears to increase with rising temperature. This indicates another suppressing effect of cyanobacteria on Daphnia fitness associated with elevated thermal conditions.

The poor manageability of filamentous or colonial cyanobacteria and the interference with the food gathering process they cause may enhance the frequency of cleaning movements of the postabdominal claws of Daphnia (Gliwicz \& Siedlar, 1980). During the cleaning of the feeding apparatus, all collected food particles (including edible ones) are discarded, thus food uptake is reduced and fewer resources are available for reproduction. This behavior also uses energy that could otherwise be allocated to reproduction. Our observations suggest that egg abortion can occur in the course of cleaning the feeding apparatus. As 
reported by Abrusán (2004), feeding disturbance caused by cyanobacterial filaments increases with rising temperature. This finding together with our observation of higher egg losses at elevated temperature supports the notion that increased abortion is associated with cleaning movements of the postabdominal claws.

In the course of this study we were unable to determine whether egg abortion was the result of eggs being lost from the brood chamber or their actual destruction. However, eggs were not observed at the bottom of the V-bottomed experimental vessels, which argues against the former. Preliminary tests with undamaged eggs (at different stages of egg development) left at the bottom of identical vessels have shown that intact eggs or "egg residues" can remain for 24-30 h (unpublished data). Despite this, the "dropping the eggs" explanation cannot be totally ruled out. It is also not possible to tell from our data, exactly when the egg abortion caused by cyanobacteria occurred. It is thought to have taken place at the beginning of the egg development period (personal observation), but further studies are required to more precisely determine the timing of this event.

Our data indicate that estimations of reproductive success in Daphnia based on female fecundity may be biased by egg abortion events caused by filamentous cyanobacteria. This phenomenon has so far not been considered in studies on interactions between Daphnia and non-toxic cyanobacteria, and it warrants further investigation. It would be particularly informative to (i) characterize the mechanism of egg abortion, (ii) determine the potential threshold filament concentration that triggers this response, and (iii) test whether the presence of other filamentous or colonial phytoplankton taxa can also result in egg abortion.

Acknowledgments We would like to thank two anonymous reviewers for critical comments which helped to improve the manuscript. The study was supported by Polish Committee for Scientific Research N N304 067236.

Open Access This article is distributed under the terms of the Creative Commons Attribution License which permits any use, distribution, and reproduction in any medium, provided the original author(s) and the source are credited.

\section{References}

Abrusán, G., 2004. Filamentous cyanobacteria, temperature and Daphnia growth: the role of fluid mechanics. Oecologia 141: 395-401.
Bednarska, A., 2006. Adaptive changes in morphology of Daphnia filter appendages in response to food stress. Polish Journal of Ecology 54: 663-667.

Bednarska, A., J. Łoś \& P. Dawidowicz, 2011. Temperature dependent effect of filamentous cyanobacteria on Daphnia magna life history traits. Journal of Limnology 70: 353-358.

Brooks, J. L., 1946. Cyclomorphosis in Daphnia. I. An analysis of D. retrocurva and D. galeata. Ecological Monographs 16: 409-447.

Claska, M. E. \& J. J. Gilbert, 1998. The effect of temperature on the response of Daphnia to toxic cyanobacterium. Freshwater Biology 39: 221-232.

Dao, T. S., L. C. Do-Hong \& C. Wiegand, 2010. Chronic effects of cyanobacterial toxins on Daphnia magna and their offspring. Toxicon 55: 1244-1254.

deBernardi, R. \& G. Giussani, 1990. Are blue-green algae a suitable food zooplankton? An overview. Hydrobiologia 200(201): 29-41.

Ghadouani, A., B. Pinel-Alloul \& E. Prepas, 2003. Effects of experimentally induced cyanobacteria blooms on crustacean zooplankton communities. Freshwater Biology 48: 363-381.

Gliwicz, Z. M. \& E. Siedlar, 1980. Food size limitation and algae interfering with food collection in Daphnia. Archiv für Hydrobiologie 88: 155-177.

Gustafsson, S., K. Rengefors \& L.-A. Hansson, 2005. Increased consumer fitness following transfer of toxin tolerance to offspring via maternal effects. Ecology 86: 2561-2567.

Kirk, K. L. \& J. J. Gilbert, 1992. Variation in herbivore response to chemical defenses: zooplankton foraging on toxic cyanobacteria. Ecology 73: 2208-2217.

Lampert, W., 1987. Laboratory studies on zooplankton-cyanobacteria interactions. New Zealand Journal of Marine and Freshwater Research 21: 483-490.

Martin-Creuzburg, D., E. Von Elert \& K. H. Hoffmann, 2008. Nutritional constraints at the cyanobacteria-Daphnia magna interface: the role of sterols. Limnology and Oceanography 53: 456-468.

Porter, K. G. \& J. D. Orcutt, 1980. Nutritional adequacy, manageability, and toxicity as factors that determine the food quality of green and blue-green algae for Daphnia. In Kerfoot, W. C. (ed.), Evolution and ecology of zooplankton communities. University Press, Hanover: 282-291.

Redfield, G. W., 1981. Nutrition and the degeneration of eggs in a limnetic daphnid. Verhandlungen der Internationalen Vereinigung für Theoretische und Angewandte Limnologie 21: 1550-1554.

Reinikainen, M., M. Ketola, M. Juntunen \& M. Walls, 1995. Effects of Microcystis aeruginosa exposure and nutritional status on the reproduction of Daphnia pulex. Journal of Plankton Research 17: 431-436.

Repka, S., 1996. Inter and intraspecific differences in Daphnia life history in response to two food sources: the green alga Scenedesmus and the filamentous cyanobacteria Oscillatoria. Journal of Plankton Research 21: 971-989.

Sperfeld, E. \& A. Wacker, 2009. Effects of temperature and dietary sterol availability on growth and cholesterol allocation of the aquatic keystone species Daphnia. Journal of Experimental Biology 212: 3051-3059.

Threlkeld, S. T., 1979. The midsummer dynamics of two Daphnia species in wintergreen lake, Michigan. Ecology 60: 165-179. 
Von Elert, E., 2004. Food quality constraints in Daphnia: interspecific differences in the response to the absence of a long chain polyunsaturated fatty acid in the food source. Hydrobiologia 526: 187-196.

Wilson, A. E., O. Sarnelle \& A. R. Tillmanns, 2006. Effects of cyanobacterial toxicity and morphology on the population growth of freshwater zooplankton: meta-analyses of laboratory experiments. Limnology and Oceanography 51: 1915-1924.

Zaffagnini, F., 1987. Reproduction in Daphnia. Memorie dell'Istituto Italiano di Idrobiologia 45: 245-284.

Zehnder, A. \& P. R. Gorham, 1960. Factors influencing the growth of Microcystis aeruginosa Kutz. emend. Elenkin. Canadian Journal of Microbiology 6: 645-660. 